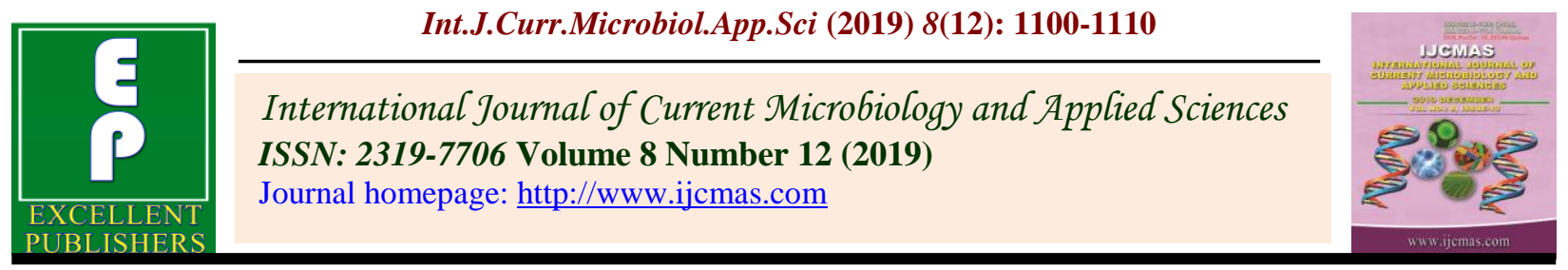

Original Research Article

https://doi.org/10.20546/ijcmas.2019.812.139

\title{
Evaluation of Staygreen Sunflower Lines for Yield under Drought Conditions (Helianthus annuus L.)
}

\author{
Harshavardan J. Hilli* and Shobha U. Immadi \\ Department of Genetics and Plant Breeding, College of Agriculture, Vijayapura, University of \\ Agricultural Sciences, Dharwad-580005, Karnataka, India \\ *Corresponding author
}

\begin{tabular}{|l|}
\hline Keyw or d s \\
Inbred lines, Stay \\
green, CMS \\
sources, Texas 16 R \\
\hline Article Info \\
\hline $\begin{array}{l}\text { Accepted: } \\
\text { 10 November } 2019 \\
\text { Available Online: } \\
\text { 10 December 2019 }\end{array}$ \\
\hline \hline
\end{tabular}

A B S T R A C T

The phenomenon of staygreen nature with desired root traits is important consideration in drought tolerance breeding. The experiment was conducted using 28 inbred lines (including 2 checks) which were developed by mutation breeding from AICRP on oilseeds, MARS, AUS, Dharwad, and on the basis of SPAD reading and stay green nature, which were essential to extract moisture from deeper layer of the soil profile. These lines were evaluated under both normal and moisture stress condition in rain out shelter to study their root characteristics and the lines that performed well were categorised as stay green lines viz DSR-13, DSR-19, DSR-23, DSR-24, DSR-37, DSR-66, DSR-107 and DSR-132 which exhibited least reduction in their yield under moisture stress condition with desired root traits. Among them DSR-37 (35.15) and DSR-132 (33.85) shown maximum root length under stress conditions, and also average yield per plant was found to be higher $(9.5 \mathrm{~g} / \mathrm{pl}$ and $8.1 \mathrm{~g} / \mathrm{pl}$ respectively) when compared to checks $95 \mathrm{C}-1$ and $6 \mathrm{D}-1$ used. These inbred lines with better performing under stress condition were further used for the development of open pollinated varieties and also used for hybridization with available CMS sources (with good combining ability) to develop drought tolerant hybrids.

\section{Introduction}

Sunflower is a cross-pollinated, annual, diploid $(2 \mathrm{x}=2 \mathrm{n}=34)$, an important edible oilseed crop ranking second after soybean in the world. The crop belongs to the family Asteraceaea, which is a native to North America. Its oil is called premium oil because of high percentage of poly-unsaturated fatty acids $(60 \%)$ including oleic acid $(16.2 \%)$ and linoleic acid $(72.5 \%)$ which helps to control cholesterol in blood (Satyabrata et al., 1988).

Sunflower is an exhaustive crop suggesting adequate water and nutrient supply which are essential factors needed for obtaining optimal 
plant growth and yield. Along with the biotic stresses like pest and diseases, an abiotic stresses like drought, temperature and sunshine affect the productivity. Drought is the most limiting of all abiotic stresses and it affects well over one-third of the soils worldwide (Skoric, 2009). Productivity of sunflower is strongly depended on availability of water and greatest yield losses occur when water shortage occurs at flowering stage (Soorninia et al., 2012). Evidence indicated that sunflower is most sensitive to water deficit stress at critical stages, flowering and seed-maturity (Chimenti et al., 2002), and drought stress during vegetative phase, flowering period and seed filling period causes considerable decrease in yield and oil content of sunflower (Ali et al., 2009).

Sunflower is more sensitive to water deficit stress at critical stages, viz., flowering and seed-maturity which causes considerable decrease in yield and oil content of sunflower (Ehab, 2006). Drought alone is responsible for 15-50 per cent reduction of the yield. Hence, it is necessary to save the yield loss due to drought in order to realize the full potential with better root performing trait (Hurd, 1974 and Richards 1993 in wheat). The root system of crop plays a very important role while considering drought tolerant breeding program. Root characteristics such as root biomass, maximum root length and the root volume helps to determine the efficiency of extraction of moisture from the soil. Sunflower genotypes having deep and extensive root system have the ability to extract water from depth of $270 \mathrm{~cm}$. Root development and growth depends upon the several factors such as plant genotype, moisture or water availability, plant population and soil condition. An increase in root length especially during the moisture stress condition along with the decrease in its lateral root length can be considered as adoptive measure for drought stress. These studies indicated that higher root growth was linked with better drought tolerance (Geeta $e t$ al., 2012) and there is a need for the development of abiotic stress tolerant hybrids and lines in order to maximize the yield.

The identification of suitable genotypes exhibiting tolerance to moisture stress as well as those showing least per cent reduction in their growth and yield attributes under soil moisture stress is necessary for the improvement of productivity under rainfed environment (Vikram et al., 2008). Hence an attempt was made with the following objective "Evaluation of stay green sunflower lines with desirable root traits for yield under drought conditions" to identify drought tolerant inbred lines.

\section{Materials and Methods}

The experimental material for the present study comprised of 28 inbred lines (including 2 checks) which were developed by mutation and hybridization breeding (involving Texas $16 \mathrm{R}$, a wild relative of cultivated sunflower which was characterised by branching and small heads but was tolerant to drought) in AICRP on oilseeds, MARS, UAS, Dharwad. From earlier studies on the basis of SPAD reading and stay green nature, they were considered as drought tolerant lines. These lines were used for the present experiment and were evaluated under both normal and moisture stress conditions in rain out shelter to study their yield and morpho-physiological traits for drought tolerance.

The experiment on evaluation of inbred lines for drought tolerance was carried out under controlled conditions in rainout shelter. Two sets of experiments in two replications were carried out. In the first set the inbred lines were evaluated under normal conditions and in the other set of experiment, the inbred lines were evaluated under drought conditions. 
In rain out shelter, the experiment was set up using PVC pipes of $1.2 \mathrm{~m}$ length which were split vertically in the middle and locking system was provided at two points along with the split end of the PVC pipes. The pipes were then put in the soil covering $2 \mathrm{ft}$ height. The pipes were filled with soil: FYM: sand in the ratio 3:2:1. Two seeds were sown per pipe and totally 28 inbred lines and two checks were used for the evaluation.

The experiment was laid out in randomized block design with two replications. In the first set of experiment, which consisted of evaluation of inbred lines under normal conditions for yield and root traits, irrigation at regular intervals was provided till maturity.

In the second set, where in the inbreds were evaluated under stress condition, normal irrigation was given until flowering stage i.e., 45-50 DAS and later on the irrigation was withheld.

\section{Management of irrigation}

The plants were irrigated alternate days for both normal and stress conditions up to flowering stage. But the irrigation was skipped to create moisture stress conditions after flowering stage. Where as the irrigation was given at normal rate upto last stage under normal conditions. During imposition of stress, water stress condition was maintained by protecting from rain under rainout shelter condition.

\section{Root sample collection}

The root samples were collected carefully by removing pipes at ground level and soaking in water for loosening of soil. Then the roots were washed thoroughly to remove all the soil particles and debris. The roots then were collected in polythene bags for collection of samples for observations. The observations viz root length, root dry weight, root fresh weight and root to shoot ratio were recorded in both the replications. Then the statistical analysis was done using windostat software.

\section{Results and Discussion}

At present drought resistant breeding in India is restricted to routine screening of varieties and hybrids for their relative drought tolerance mostly by field screening but identification of potential sources of drought tolerance is critical to undertake breeding programme. Hence an attempt was made to screen the sunflower inbred lines with better root system and high WUE which can perform well under water limited conditions and also to identify the physiological traits, related to drought tolerance. Analysis of variance (Table 1) revealed that there was significant difference observed among the genotypes for all the traits (Plant height, SPAD at 45 DAS, SPAD at 60 DAS, days to flowering, days to maturity, head diameter, relative water content, test weight, seed yield per plant, root length, root dry weight, root fresh weight and root to shoot ratio) at $5 \%$ significance level under different soil moisture regimes. Mean performance of all gentypes is shown in Table 2. Among the 28 inbreds evaluated, eight lines shown (Table 3 ) superior performance under both the normal and stress conditions with respect to SPAD readings at $60 \mathrm{DAS}$, root length, relative water content, root to shoot ratio and also yield per plant. DSR-37 and DSR-132 inbreds have shown better performance with respect to root trait i.e $35.15 \mathrm{~cm}$ and $33.85 \mathrm{~cm}$ of root length (Increase in root length in stress condition is an adaptive mechanism for drought tolerant genotypes. Therefore higher value may be used for the discrimination between drought tolerant and susceptible genotypes). Rauf and Sadqat (2008) stated that increase in root length occurred due to higher osmotic adjustment ability of drought genotypes (Fig. $1)$. 
Table.1 Analysis of variance for 13 different traits under normal and moisture stress condition in sunflower

\begin{tabular}{|c|c|c|c|c|c|c|c|c|c|c|c|c|c|c|c|}
\hline \multirow{2}{*}{$\begin{array}{l}\text { Source of } \\
\text { variation }\end{array}$} & \multirow[t]{2}{*}{ df } & \multicolumn{2}{|c|}{ SPAD at 45 DAS } & \multicolumn{2}{|c|}{ SPAD at 60 DAS } & \multicolumn{2}{|c|}{ DFF } & \multicolumn{2}{|c|}{ DM } & \multicolumn{2}{|c|}{ PH (cm) } & \multicolumn{2}{|c|}{ RWC (\%) } & \multicolumn{2}{|c|}{ HD (cm) } \\
\hline & & $\mathbf{N}$ & $\mathbf{S}$ & $\mathbf{N}$ & $\mathbf{S}$ & $\mathbf{N}$ & $\mathbf{S}$ & $\mathbf{N}$ & $\mathbf{S}$ & $\mathbf{N}$ & $\mathbf{S}$ & $\mathbf{N}$ & $\mathbf{S}$ & $\mathbf{N}$ & $\mathbf{S}$ \\
\hline Replication & 1 & 4.79 & 17.55 & 35.50 & 17.52 & 62.02 & 45.07 & 58.02 & 1.07 & 180.27 & 39.20 & 5.13 & 10.37 & 0.46 & 0.39 \\
\hline Treatment & 29 & $58.13 *$ & $62.43 *$ & $33.34 *$ & $38.21 *$ & $98.47 *$ & $93.37 *$ & $40.74 *$ & $69.16^{*}$ & $198.75^{*}$ & $150.79 *$ & $70.75 *$ & $140.20 *$ & $6.43 *$ & $8.00 *$ \\
\hline Error & 29 & 7.57 & 9.13 & 7.41 & 8.89 & 38.71 & 32.41 & 16.60 & 15.86 & 28.65 & 23.89 & 10.10 & 8.32 & 0.57 & 0.56 \\
\hline
\end{tabular}

\begin{tabular}{|c|c|c|c|c|c|c|c|c|c|c|c|c|c|}
\hline \multirow{2}{*}{$\begin{array}{l}\text { Source of } \\
\text { variation }\end{array}$} & \multirow[t]{2}{*}{ df } & \multicolumn{2}{|c|}{ RL (cm) } & \multicolumn{2}{|c|}{ FWR (g) } & \multicolumn{2}{|c|}{ DWR（g) } & \multicolumn{2}{|c|}{ Root/shoot ratio } & \multicolumn{2}{|c|}{ Test weight (g) } & \multicolumn{2}{|c|}{ Yield/plant (g) } \\
\hline & & $\mathbf{N}$ & $\mathbf{S}$ & $\mathbf{N}$ & $\mathbf{S}$ & $\mathbf{N}$ & $\mathbf{S}$ & $\mathbf{N}$ & $\mathbf{S}$ & $\mathbf{N}$ & $\mathbf{S}$ & $\mathbf{N}$ & $\mathbf{S}$ \\
\hline Replication & 1 & 10.58 & 8.82 & 1.53 & 1.22 & 1.22 & 0.12 & 0.0001 & 0.0010 & 0.16 & 0.36 & 0.01 & 0.20 \\
\hline Treatment & 29 & $55.41 *$ & $49.96 *$ & $4.65^{*}$ & $5.37 *$ & $1.97 *$ & $4.54 *$ & $0.01 *$ & $0.01 *$ & $1.63 *$ & $2.34 *$ & $5.45^{*}$ & $7.34 *$ \\
\hline Error & 29 & 2.98 & 4.76 & 0.33 & 0.46 & 0.26 & 0.26 & 0.0016 & 0.0022 & 0.73 & 0.92 & 0.71 & 0.70 \\
\hline
\end{tabular}

* Significance at 5\%; DFF- Days to $50 \%$ flowering, DM- Days to maturity, PH- Plant height, RWC- Relative water content, HD- Head diameter, RL- Root length, FWRFresh weight of roots, DWR- Dry weight of roots

Table.2 Mean and range for 13 different traits in normal and moisture stress condition in sunflower

\begin{tabular}{|c|c|c|c|c|}
\hline \multirow[t]{2}{*}{ Characters } & \multicolumn{2}{|c|}{ Range } & \multicolumn{2}{|c|}{ Mean } \\
\hline & Normal & Stress & Normal & Stress \\
\hline SPAD@45DAS & $38.88-60.18$ & $33.78-58.53$ & 46.15 & 43.86 \\
\hline SPAD@60 DAS & $31.33-46.95$ & $27.18-48.15$ & 38.10 & 37.14 \\
\hline DFF (days) & $52.00-77.00$ & $52.00-74.00$ & 63.82 & 62.43 \\
\hline RWC (\%) & $37.90-60.60$ & $30.25-57.10$ & 50.46 & 43.33 \\
\hline Plant height $(\mathrm{cm})$ & $36.25-67.75$ & $40.50-65.75$ & 53.54 & 51.22 \\
\hline Days to maturity & $89.00-108.00$ & $81.00-95.00$ & 98.64 & 88.00 \\
\hline Head diameter $(\mathrm{cm})$ & $3.75-9.25$ & $3.25-8.80$ & 6.18 & 5.47 \\
\hline Root length $(\mathrm{cm})$ & $10.93-34.90$ & $12.83-35.15$ & 17.20 & 18.51 \\
\hline Fresh weight of roots (g) & $3.33-8.68$ & $3.89-9.53$ & 5.72 & 6.21 \\
\hline Dry weight of roots (g) & $3.05-6.98$ & $2.18-6.95$ & 4.48 & 4.35 \\
\hline Root : Shoot ratio & $0.22-0.53$ & $0.28-0.55$ & 0.33 & 0.37 \\
\hline Test weight (g) & $2.95-4.8$ & $2.71-4.48$ & 3.81 & 3.36 \\
\hline Seed yield/plant (g) & $4.20-10.10$ & $2.70-9.50$ & 6.34 & 5.37 \\
\hline
\end{tabular}


Table.3 Top eight inbreds selected based on per se performance and per cent reduction under stress condition for 7 different traits in sunflower

\begin{tabular}{|c|c|c|c|c|c|c|c|c|c|c|c|c|c|c|}
\hline \multirow[t]{2}{*}{ Inbreds } & \multicolumn{2}{|c|}{$\begin{array}{c}\text { SPAD AT } 60 \\
\text { DAS }\end{array}$} & \multicolumn{2}{|c|}{$\begin{array}{l}\text { Plant height } \\
\text { (cm) }\end{array}$} & \multicolumn{2}{|c|}{$\begin{array}{c}\text { RWC } \\
(\%)\end{array}$} & \multicolumn{2}{|c|}{$\begin{array}{l}\text { Root length } \\
(\mathrm{cm})\end{array}$} & \multicolumn{2}{|c|}{$\begin{array}{c}\text { Root }: \text { shoot } \\
\text { ratio }\end{array}$} & \multicolumn{2}{|c|}{$\begin{array}{l}\text { Test weight } \\
\text { (g) }\end{array}$} & \multicolumn{2}{|c|}{$\begin{array}{l}\text { Seed yl/pl } \\
(\mathrm{g})\end{array}$} \\
\hline & $\mathbf{N}$ & $\mathbf{S}$ & $\mathbf{N}$ & $\mathbf{S}$ & $\mathbf{N}$ & $\mathbf{S}$ & $\mathbf{N}$ & $\mathbf{S}$ & $\mathbf{N}$ & $\mathbf{S}$ & $\mathbf{N}$ & $\mathbf{S}$ & $\mathbf{N}$ & $\mathbf{S}$ \\
\hline DSR-13 & 38.53 & 40.24 & 65.50 & 62.75 & 55.58 & 51.08 & 16.38 & 19.88 & 0.25 & 0.32 & 4.10 & 3.87 & 8.70 & 8.20 \\
\hline DSR-19 & 42.93 & 41.14 & 55.00 & 51.00 & 49.80 & 46.78 & 14.43 & 18.43 & 0.27 & 0.37 & 4.02 & 3.94 & 8.80 & 8.50 \\
\hline DSR-23 & 37.80 & 40.01 & 65.50 & 64.25 & 52.28 & 49.28 & 16.55 & 20.55 & 0.23 & 0.32 & 4.11 & 3.89 & 8.40 & 8.10 \\
\hline DSR-35 & 35.55 & 35.76 & 65.25 & 63.00 & 55.08 & 54.88 & 16.03 & 20.03 & 0.25 & 0.32 & 4.33 & 4.05 & 8.40 & 8.10 \\
\hline DSR-37 & 44.43 & 43.93 & 66.25 & 63.50 & 58.75 & 54.25 & 34.90 & 35.15 & 0.53 & 0.55 & 4.80 & 4.34 & 10.10 & 9.50 \\
\hline DSR-66 & 46.95 & 48.15 & 57.25 & 53.25 & 55.53 & 50.53 & 19.40 & 20.90 & 0.34 & 0.40 & 4.78 & 4.48 & 8.10 & 7.10 \\
\hline DSR-107 & 43.03 & 39.73 & 64.25 & 60.25 & 55.13 & 51.13 & 18.18 & 22.18 & 0.28 & 0.37 & 3.90 & 3.12 & 7.30 & 7.50 \\
\hline DSR-132 & 42.63 & 41.88 & 67.75 & 65.75 & 55.65 & 49.20 & 33.50 & 33.85 & 0.49 & 0.51 & 4.34 & 4.08 & 9.11 & 8.10 \\
\hline RHA 95 C-1 & 38.25 & 39.38 & 50.50 & 46.50 & 58.75 & 54.75 & 15.28 & 17.18 & 0.30 & 0.38 & 3.23 & 2.88 & 6.10 & 5.10 \\
\hline RHA 6D-1 & 41.40 & 35.90 & 60.00 & 56.00 & 51.30 & 54.80 & 14.90 & 16.30 & 0.25 & 0.29 & 3.80 & 3.23 & 6.20 & 5.70 \\
\hline Mean & 41.19 & 40.51 & 61.75 & 58.80 & 55.66 & 52.39 & 19.22 & 21.71 & 0.31 & 0.37 & 3.81 & 3.36 & 7.94 & 7.36 \\
\hline SEm \% & 1.92 & 2.11 & 3.78 & 3.46 & 2.25 & 2.04 & 1.22 & 1.54 & 0.03 & 0.03 & 0.45 & 0.43 & 0.59 & 0.59 \\
\hline CD@5\% & 5.57 & 6.10 & 10.95 & 10.00 & 6.50 & 5.90 & 3.53 & 4.46 & 0.08 & 0.10 & 1.91 & 1.82 & 1.72 & 1.71 \\
\hline CV \% & 7.13 & 8.05 & 10.03 & 9.58 & 6.27 & 6.61 & 10.32 & 11.58 & 12.69 & 12.72 & 13.84 & 14.22 & 13.21 & 15.27 \\
\hline
\end{tabular}

DFF- Days to $50 \%$ flowering, DM- Days to maturity, PH- Plant height, RWC- Relative water content, HD- Head diameter, RL- Root length, FWR- Fresh weight of roots, DWR- Dry weight of roots, TW- Test weight, Yl/Pl- Yield per plant 
Table.4 Classification of inbreds as low and high performing types based on 6 drought related traits under moisture stress condition in sunflower

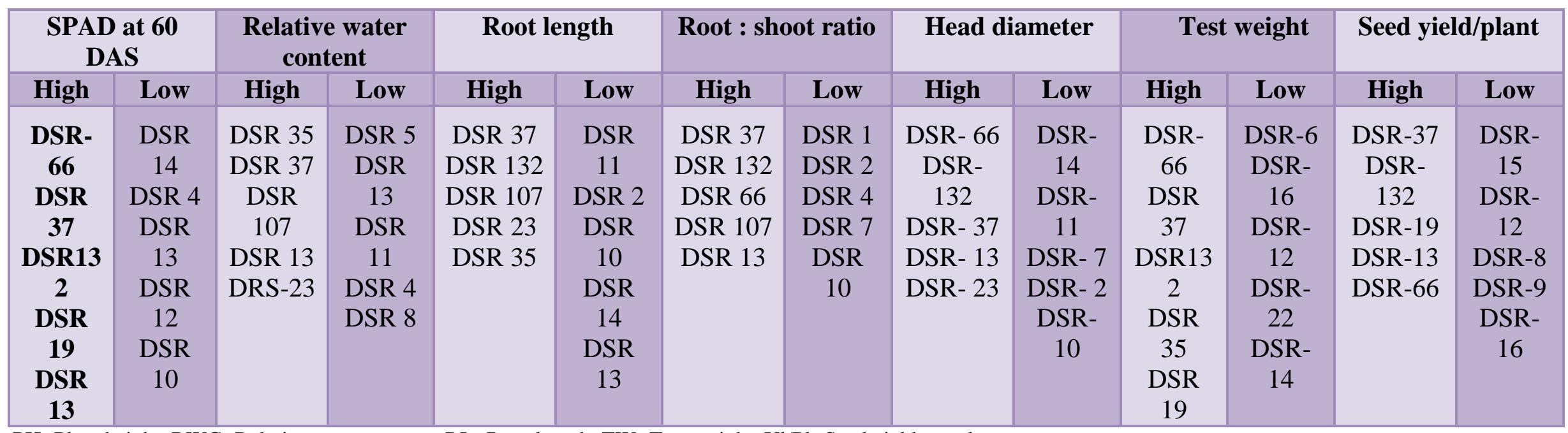

PH- Plant height, RWC- Relative water content, RL- Root length, TW- Test weight, Yl/Pl- Seed yield per plant 
Table.5 Per cent reduction in the per se performance for 13 different traits under moisture stress conditions in sunflower

\begin{tabular}{|c|c|c|c|c|c|c|c|c|c|c|c|c|c|c|}
\hline $\begin{array}{l}\text { SI. } \\
\text { No. }\end{array}$ & Inbreds & $\begin{array}{c}\text { SPAD @ } \\
45 \text { DAS }\end{array}$ & $\begin{array}{r}\text { SPAD } \\
\text { @ } 60 \\
\text { DAS }\end{array}$ & DFF & DM & PH & RWC & HD & RL & FWR & DWR & $\mathbf{R} / \mathbf{S}$ & Tw (g) & $\mathbf{Y l} / \mathbf{P I}$ \\
\hline 1 & DSR-13 & 7.47 & -4.44 & 3.90 & 10.28 & 4.20 & 8.10 & 5.81 & -21.37 & -3.11 & 4.73 & -28.00 & 5.61 & 5.75 \\
\hline 2 & DSR-1 & 1.49 & 0.64 & 0.00 & -3.16 & 7.37 & 7.51 & 36.90 & -20.87 & -15.63 & -26.36 & -29.17 & 12.97 & 4.69 \\
\hline 3 & DSR-17 & -1.32 & 6.55 & 0.00 & 2.02 & -3.07 & 2.20 & 20.00 & -14.07 & -6.33 & 15.48 & -10.00 & 11.43 & 43.55 \\
\hline 4 & DSR-2 & 17.52 & -7.11 & 0.00 & -4.12 & -5.63 & 9.93 & 34.45 & -14.61 & -25.53 & 18.99 & -3.33 & 16.01 & 23.73 \\
\hline 5 & DSR-19 & 2.65 & 4.17 & 4.30 & -2.02 & 7.27 & 6.02 & 9.41 & -27.72 & -19.87 & -6.69 & -37.04 & 1.99 & 3.41 \\
\hline 6 & DSR-16 & -9.03 & 7.01 & 0.00 & 1.06 & 0.59 & 22.78 & 5.80 & -17.58 & 6.45 & 42.92 & -25.81 & 6.45 & 4.76 \\
\hline 7 & DSR-3 & 5.09 & -1.02 & 0.00 & -4.00 & 6.75 & 15.13 & 24.81 & -13.15 & -30.03 & 26.89 & -21.43 & 10.96 & 28.81 \\
\hline 8 & DSR-23 & 6.44 & -5.85 & 4.10 & 2.02 & 3.38 & 5.74 & 3.64 & -24.17 & -22.39 & 0.66 & -39.13 & 5.28 & 3.57 \\
\hline 9 & DSR-15 & 12.61 & 1.74 & 0.00 & 1.08 & 8.70 & 24.48 & 15.56 & 1.12 & -7.07 & 22.35 & -7.69 & 17.55 & 14.29 \\
\hline 10 & DSR-4 & 5.22 & -7.09 & 2.80 & -3.88 & 7.17 & 31.32 & 26.09 & -15.69 & -31.91 & 13.68 & -24.00 & 11.31 & 24.56 \\
\hline 11 & DSR-14 & 10.03 & 13.25 & 0.00 & 1.00 & 4.14 & 6.59 & 27.78 & -5.76 & -7.88 & 24.00 & -9.09 & 15.53 & 4.08 \\
\hline 12 & DSR-35 & -9.95 & 3.53 & 4.00 & 1.06 & 3.45 & 2.18 & 2.70 & -24.95 & -11.72 & -0.60 & -28.00 & 6.47 & 3.57 \\
\hline 13 & DSR-5 & 2.38 & 18.15 & 4.90 & -3.00 & 6.59 & 30.86 & 19.80 & -9.52 & -22.48 & 18.66 & -15.15 & 15.48 & 23.08 \\
\hline 14 & DSR-6 & 4.94 & -9.99 & -5.70 & -2.88 & -4.14 & 28.27 & 22.68 & -10.70 & -15.22 & -7.62 & -7.14 & 16.10 & 18.75 \\
\hline 15 & DSR-12 & 11.23 & 17.34 & -1.40 & 1.01 & 1.16 & 32.96 & 13.48 & -3.86 & -9.89 & 22.22 & -5.56 & 14.04 & 40.00 \\
\hline 16 & DSR-37 & 1.75 & 1.13 & 5.50 & 4.44 & 4.15 & 7.66 & 4.62 & -0.72 & -15.57 & -8.76 & -3.77 & 9.58 & 5.94 \\
\hline 17 & DSR-18 & 0.00 & 7.71 & 0.00 & 0.97 & 0.00 & 19.03 & 13.33 & -18.36 & -14.17 & 8.45 & -17.24 & 4.75 & 22.00 \\
\hline 18 & DSR-7 & 4.67 & -3.98 & 3.50 & -3.03 & 8.12 & 31.06 & 25.00 & -3.43 & -20.00 & 30.00 & -10.81 & 13.33 & 9.43 \\
\hline 19 & DSR-8 & 0.21 & 6.67 & 3.30 & -2.88 & 7.24 & 15.59 & 17.17 & -12.70 & -21.09 & 26.91 & -21.43 & 16.50 & 23.40 \\
\hline 20 & DSR-66 & 1.18 & -2.56 & 5.30 & 2.11 & 6.99 & 9.00 & -0.57 & -7.73 & -14.98 & -5.07 & -17.65 & 6.28 & 12.35 \\
\hline 21 & DSR-125 & 6.54 & 1.76 & 4.70 & 0.99 & 5.90 & 4.31 & 8.57 & -21.65 & -13.75 & -13.16 & -29.63 & 11.64 & 7.69 \\
\hline 22 & DSR-107 & 2.74 & 7.67 & 4.90 & -1.06 & 6.23 & 7.26 & 8.89 & -22.00 & -24.74 & -31.58 & -32.14 & 20.00 & 6.16 \\
\hline 23 & DSR-9 & 10.73 & 8.79 & 2.90 & -0.93 & 7.80 & 21.91 & 1.11 & -13.35 & -4.67 & 27.61 & -23.08 & 22.25 & 22.92 \\
\hline 24 & DSR-10 & 14.22 & 6.08 & -1.90 & 1.00 & -15.86 & 24.56 & 2.50 & -14.75 & -9.80 & 30.53 & 7.89 & 9.91 & 35.59 \\
\hline 25 & DSR-11 & 1.44 & -8.45 & 0.00 & 1.06 & 7.43 & 28.96 & 2.67 & -17.38 & -4.74 & 45.50 & -28.00 & 14.32 & 23.08 \\
\hline 26 & DSR-132 & 10.93 & 5.98 & 5.20 & 1.12 & 2.95 & 6.74 & 5.95 & -1.04 & -10.00 & -18.07 & -4.08 & 5.99 & 10.99 \\
\hline 27 & $95 \mathrm{C}-1$ & 3.03 & -2.61 & 5.30 & 4.08 & 8.82 & 6.81 & 3.57 & -12.43 & -17.27 & 1.08 & -26.67 & 10.84 & 16.39 \\
\hline 28 & $6 \mathrm{D}-1$ & 5.83 & 13.29 & 0.00 & 1.00 & 6.67 & 1.93 & 8.40 & -9.40 & -5.68 & 8.69 & -16.00 & 15.00 & 8.06 \\
\hline
\end{tabular}


Table.6 The per se performance of inbred lines for 13 different traits under normal and stress conditions in sunflower

\begin{tabular}{|c|c|c|c|c|c|c|c|c|c|c|c|c|c|}
\hline \multirow[t]{2}{*}{ Sl.No. } & \multirow[t]{2}{*}{ Inbreds } & \multicolumn{2}{|c|}{ SPAD @45DAS } & \multicolumn{2}{|c|}{ SPAD@60 DAS } & \multicolumn{2}{|c|}{ DFF } & \multicolumn{2}{|c|}{ DM } & \multicolumn{2}{|c|}{ PH (cm) } & \multicolumn{2}{|c|}{ RWC (\%) } \\
\hline & & $\mathbf{N}$ & $\mathbf{S}$ & $\mathbf{N}$ & $\mathbf{S}$ & $\mathbf{N}$ & $\mathbf{S}$ & $\mathrm{N}$ & $\mathrm{S}$ & $\mathrm{N}$ & S & $\mathbf{N}$ & $\mathbf{S}$ \\
\hline 1 & DSR-13 & 44.18 & 40.88 & 38.53 & 40.24 & 77.00 & $74.00 *$ & $107.00 *$ & $92.00 *$ & $66.50 *$ & $62.75 *$ & $55.58 *$ & $51.08 *$ \\
\hline 2 & DSR-1 & 40.18 & 39.58 & 36.13 & 35.90 & 73.00 & $73.00 *$ & 95.00 & $89.00 *$ & 54.25 & 50.25 & 46.58 & 43.08 \\
\hline 3 & DSR-17 & 45.50 & 46.10 & 40.45 & 37.80 & 66.00 & $66.00 *$ & 99.00 & $92.00 *$ & 40.75 & 42.00 & 45.38 & 44.38 \\
\hline 4 & DSR-2 & 49.08 & 40.48 & 33.03 & 35.38 & $65.00 *$ & $65.00 *$ & 97.00 & $89.00 *$ & 40.00 & 42.25 & 45.30 & 40.80 \\
\hline 5 & DSR-19 & 49.08 & 47.78 & $42.93 *$ & 41.14 & $69.00 *$ & $66.00 *$ & 99.00 & $86.00 *$ & $55.00 *$ & 51.00 & 49.80 & 46.80 \\
\hline 6 & DSR-16 & 39.88 & 43.48 & 37.83 & 35.18 & 62.00 & 62.00 & 94.00 & $88.00 *$ & 42.25 & 42.00 & 46.10 & 35.60 \\
\hline 7 & DSR-3 & 41.23 & 39.13 & 34.18 & 34.53 & $69.00 *$ & 69.00 & 100.00 & $89.00 *$ & $59.25 *$ & $55.25 *$ & 46.28 & 39.28 \\
\hline 8 & DSR-23 & 43.45 & 40.65 & 37.80 & 40.01 & $73.00 *$ & $70.00 *$ & 99.00 & $91.00 *$ & $66.50 *$ & 64.25 & $52.28 *$ & $49.28 *$ \\
\hline 9 & DSR-15 & 46.80 & 40.90 & 37.25 & 36.60 & 57.00 & 57.00 & 93.00 & 84.00 & 46.00 & 42.00 & 46.98 & 35.48 \\
\hline 10 & DSR-4 & 40.20 & 38.10 & 33.15 & 35.50 & $71.00 *$ & $69.00 *$ & $103.0 *$ & $91.00 *$ & 55.75 & 51.75 & 51.08 & 35.08 \\
\hline 11 & DSR-14 & 38.88 & 34.98 & 31.33 & 27.18 & 58.00 & 56.00 & $100.0^{*}$ & $90.00 *$ & 42.25 & 40.50 & 37.95 & 35.45 \\
\hline 12 & DSR-35 & 42.20 & 46.40 & 36.55 & 35.26 & $75.00 *$ & $72.00 *$ & 94.00 & 83.00 & $65.25 *$ & $63.00 *$ & $55.08 *$ & $53.88 *$ \\
\hline 13 & DSR-5 & 46.18 & 45.08 & 36.63 & 29.98 & 61.00 & 58.00 & $100.0^{*}$ & $85.00 *$ & 45.50 & 42.50 & 43.75 & 30.25 \\
\hline 14 & DSR-6 & 52.60 & 50.00 & 33.55 & 36.90 & 57.00 & 56.00 & $104.0 *$ & 91.00 & 42.25 & 44.00 & 49.53 & 35.53 \\
\hline 15 & DSR-12 & 45.40 & 40.30 & 38.35 & 31.70 & $72.00 *$ & $73.00 *$ & 99.00 & 90.00 & 43.25 & 42.75 & $57.65 *$ & 38.65 \\
\hline 16 & DSR-37 & 51.43 & 50.53 & $44.43 *$ & $43.93 *$ & 55.00 & 52.00 & 90.00 & 80.00 & $66.25 *$ & $63.50 *$ & $58.75 *$ & $54.25 *$ \\
\hline 17 & DSR-18 & 44.18 & 40.88 & 38.53 & 40.24 & $77.00 *$ & $74.00^{*}$ & $107.0^{*}$ & $93.00 *$ & $65.50 *$ & $62.75 *$ & $55.58 *$ & $51.08 *$ \\
\hline 18 & DSR-7 & 44.93 & 42.83 & 33.88 & 35.23 & 57.00 & 55.00 & 99.00 & $90.00 *$ & 49.25 & 45.25 & 53.13 & 36.63 \\
\hline 19 & DSR-8 & 46.80 & 46.70 & 39.75 & 37.10 & 60.00 & 58.00 & $104.0 *$ & $89.00 *$ & $55.25 *$ & 51.25 & 41.70 & 35.20 \\
\hline 20 & DSR-66 & $55.10 *$ & $54.45 *$ & $46.95 *$ & $48.15 *$ & 57.00 & 54.00 & 95.00 & $86.00 *$ & $57.25 *$ & 53.25 & $55.53 *$ & $50.53 *$ \\
\hline 21 & DSR-125 & 55.80 & $52.15 *$ & $42.63 *$ & 41.88 & 64.00 & 61.00 & $101.0 *$ & 83.00 & $67.75 *$ & $63.75 *$ & 50.70 & $48.51 *$ \\
\hline 22 & DSR-107 & $60.18 *$ & $58.53 *$ & $43.03 *$ & 39.73 & 61.00 & 58.00 & 94.00 & 83.00 & $64.25^{*}$ & $60.25 *$ & $55.13 *$ & $51.13 *$ \\
\hline 23 & DSR-9 & 42.88 & 38.28 & 35.83 & 32.68 & $69.00 *$ & $67.00 *$ & 108.00 & $95.00 *$ & 51.25 & 47.25 & 45.65 & 35.65 \\
\hline 24 & DSR-10 & 39.38 & 33.78 & 35.38 & 33.23 & 58.00 & 57.00 & 100.00 & $91.00 *$ & 36.25 & 42.00 & $52.93 *$ & 39.93 \\
\hline 25 & DSR-11 & 41.78 & 41.18 & 33.73 & 36.58 & 64.00 & $64.00 *$ & 94.00 & $87.00 *$ & 43.75 & 40.50 & 48.35 & 34.35 \\
\hline 26 & DSR-132 & 52.63 & 46.88 & $45.15 *$ & $42.45 *$ & 58.00 & 55.00 & 89.00 & 81.00 & $67.75 *$ & $65.75 *$ & $55.65 *$ & $51.90 *$ \\
\hline 27 & $95 \mathrm{C}-1$ & 43.88 & 42.55 & 38.38 & 39.38 & 57.00 & 54.00 & 98.00 & $90.00 *$ & 51.00 & 46.50 & $58.75 *$ & $54.75 *$ \\
\hline \multirow[t]{5}{*}{28} & $6 \mathrm{D}-1$ & 48.40 & 45.58 & $41.40 *$ & 35.90 & 57.00 & 57.00 & $100.0 *$ & $86.00 *$ & $60.00 *$ & $56.00 *$ & 51.80 & $50.80 *$ \\
\hline & MEAN & 46.15 & 43.86 & 38.10 & 37.14 & 63.82 & 62.43 & 98.64 & 88.00 & 53.54 & 51.22 & 50.46 & 43.33 \\
\hline & SEm \% & 1.95 & 2.14 & 1.92 & 2.11 & 4.4 & 4.03 & 2.88 & 2.82 & 3.78 & 3.46 & 2.25 & 2.04 \\
\hline & $\mathrm{CD}$ at $5 \%$ & 5.63 & 6.18 & 5.57 & 6.10 & 12.72 & 11.64 & 8.33 & 8.14 & 10.95 & 10 & 6.50 & 5.90 \\
\hline & CV \% & 5.95 & 6.85 & 7.13 & 8.05 & 9.89 & 9.25 & 4.14 & 4.08 & 10.03 & 9.58 & 6.27 & 6.61 \\
\hline
\end{tabular}




\begin{tabular}{|c|c|c|c|c|c|c|c|c|c|c|c|c|c|c|c|}
\hline \multirow[t]{2}{*}{ Sl.No. } & \multirow[t]{2}{*}{ INBREDS } & \multicolumn{2}{|c|}{ HD (cm) } & \multicolumn{2}{|c|}{ RL (cm) } & \multicolumn{2}{|c|}{ FWR(g) } & \multicolumn{2}{|c|}{ DWR (g) } & \multicolumn{2}{|c|}{ R:S ratio } & \multicolumn{2}{|c|}{ TW (g) } & \multicolumn{2}{|c|}{ Seed Yl/pl (g) } \\
\hline & & $\mathbf{N}$ & S & $\mathbf{N}$ & $\mathbf{S}$ & $\mathbf{N}$ & S & $\mathbf{N}$ & $\mathbf{S}$ & $\mathbf{N}$ & $\mathbf{S}$ & $\mathbf{N}$ & $\mathbf{S}$ & $\mathbf{N}$ & S \\
\hline 1 & DSR-13 & $8.60 *$ & $8.10 *$ & 16.38 & 19.88 & $8.68 *$ & $8.95 *$ & $6.98 *$ & $6.65 *$ & 0.25 & 0.32 & $4.10 *$ & $3.87 *$ & 8.70 & $8.20 *$ \\
\hline 2 & DSR-1 & $8.40 *$ & 5.30 & 12.70 & 15.35 & $8.00 *$ & $9.25 *$ & 5.50 & $6.95 *$ & 0.24 & 0.31 & $3.70 *$ & $3.22 *$ & 6.00 & 5.10 \\
\hline 3 & DSR-17 & 5.00 & 4.00 & 16.35 & 18.65 & 5.88 & 5.53 & 3.93 & 4.65 & $0.44 *$ & 0.40 & $3.50 *$ & $3.10 *$ & 6.20 & 3.50 \\
\hline 4 & DSR-2 & 5.95 & 3.90 & 13.10 & 11.43 & 4.70 & 5.90 & 3.95 & 3.20 & 0.30 & 0.31 & $3.56 *$ & $2.99 *$ & 5.90 & 4.50 \\
\hline 5 & DSR-19 & $8.50 *$ & $7.70 *$ & 14.43 & 18.43 & 6.04 & 7.24 & 4.78 & 5.10 & 0.27 & 0.37 & $4.02 *$ & $3.94 *$ & 8.80 & $8.50 *$ \\
\hline 6 & DSR-16 & 4.25 & 4.00 & 15.38 & 13.08 & 5.08 & 5.43 & 4.87 & 2.78 & 0.39 & 0.31 & 3.10 & $2.90 *$ & 4.20 & 4.00 \\
\hline 7 & DSR-3 & 6.65 & 5.00 & 16.35 & 18.50 & 3.33 & 4.33 & 3.05 & 2.23 & 0.28 & 0.34 & $3.56 *$ & $3.17 *$ & 5.90 & 4.20 \\
\hline 8 & DSR-23 & $8.25 *$ & $7.95 *$ & 16.55 & 20.55 & 5.36 & 6.56 & 4.56 & 4.53 & 0.23 & 0.32 & $4.11 *$ & $3.89 *$ & 8.40 & $8.00 *$ \\
\hline 9 & DSR-15 & 4.50 & 3.80 & 17.80 & 17.60 & 4.95 & 5.30 & 4.25 & 3.30 & 0.39 & 0.42 & $3.76 *$ & $3.10 *$ & 4.20 & 3.60 \\
\hline 10 & DSR-4 & 5.75 & 4.25 & 15.85 & 13.70 & 5.58 & 4.23 & 3.28 & 3.80 & 0.31 & 0.25 & $3.89 *$ & $3.45 *$ & 5.70 & 4.30 \\
\hline 11 & DSR-14 & 4.50 & 3.25 & 13.90 & 14.70 & 4.44 & 4.79 & 4.00 & 3.04 & 0.33 & 0.36 & $3.67 *$ & $3.10 *$ & 4.90 & 4.70 \\
\hline 12 & DSR-35 & 7.25 & $7.05 *$ & 16.03 & 20.03 & 6.57 & 7.34 & 4.97 & 5.00 & 0.25 & 0.32 & $4.33 *$ & $4.05 *$ & 8.40 & $8.10 *$ \\
\hline 13 & DSR-5 & 5.05 & 4.05 & 14.70 & 16.10 & 4.67 & 5.72 & 4.18 & 3.40 & 0.33 & 0.38 & $4.07 *$ & $3.44 *$ & 5.20 & 4.00 \\
\hline 14 & DSR-6 & 4.85 & 3.75 & 19.65 & 17.75 & $7.95 *$ & 6.90 & $5.65 *$ & 5.25 & $0.45 *$ & 0.42 & $3.23 *$ & $2.71 *$ & 4.80 & 3.90 \\
\hline 15 & DSR-12 & 4.45 & 3.85 & 15.55 & 16.15 & 3.54 & 3.89 & 3.06 & 2.38 & 0.36 & 0.38 & $3.56 *$ & $3.06 *$ & 4.50 & 2.70 \\
\hline 16 & DSR-37 & $8.65 *$ & $8.25 *$ & $34.90 *$ & $35.15^{*}$ & $8.03 *$ & $9.28 *$ & $6.28 *$ & $6.83 *$ & $0.53 *$ & $0.55 *$ & $4.80 *$ & $4.34 *$ & $10.10 *$ & $9.50 *$ \\
\hline 17 & DSR-18 & 4.50 & 3.90 & 12.53 & 14.83 & 3.81 & 4.35 & 3.55 & 3.25 & 0.29 & 0.34 & 2.95 & $2.81 *$ & 5.00 & 3.90 \\
\hline 18 & DSR-7 & 5.00 & 3.75 & 18.70 & 18.08 & 4.80 & 4.00 & 2.59 & 3.70 & $0.41 *$ & 0.37 & $3.60 *$ & $3.12 *$ & 5.30 & 4.80 \\
\hline 19 & DSR-8 & 4.95 & 4.10 & 15.35 & 17.30 & 4.03 & 4.88 & 3.53 & 2.58 & 0.28 & 0.34 & $4.00 *$ & $3.34 *$ & 4.70 & 3.60 \\
\hline 20 & DSR-66 & $8.75 *$ & $8.80 *$ & 19.40 & 20.90 & $8.01 *$ & $9.21 *$ & $6.31 *$ & $6.63 *$ & 0.34 & 0.40 & $4.78 *$ & $4.48 *$ & 8.10 & 7.10 \\
\hline 21 & DSR-125 & 7.00 & 6.40 & 18.48 & 22.48 & 6.18 & 7.03 & 4.18 & 4.73 & 0.27 & 0.35 & $3.78 *$ & $3.34 *$ & 7.80 & 7.20 \\
\hline 22 & DSR-107 & 6.75 & 6.15 & 18.18 & 22.18 & 4.85 & 6.05 & 3.61 & 4.75 & 0.28 & 0.37 & $3.90 *$ & $3.12 *$ & 7.30 & 6.60 \\
\hline 23 & DSR-9 & 4.50 & 4.45 & 19.85 & 22.50 & 5.78 & 6.05 & 5.18 & 3.75 & 0.39 & 0.48 & $4.00 *$ & $3.11 *$ & 4.80 & 3.70 \\
\hline 24 & DSR-10 & 4.00 & 3.90 & 14.78 & 12.88 & 5.60 & 5.10 & 3.30 & 4.75 & 0.35 & 0.38 & $3.43 *$ & $3.09 *$ & 5.90 & 3.80 \\
\hline 25 & DSR-11 & 3.75 & 3.65 & 10.93 & 12.83 & 4.22 & 4.42 & 4.00 & 2.18 & 0.25 & 0.32 & $3.77 *$ & $3.23 *$ & 5.20 & 4.00 \\
\hline 26 & DSR-132 & $9.25 *$ & $8.70 *$ & 33.50 * & $33.85^{*}$ & $8.50 *$ & $9.35 *$ & $5.70 *$ & $6.73 *$ & $0.49 *$ & $0.51 *$ & $4.34 *$ & $4.08 *$ & $9.10 *$ & $8.10 *$ \\
\hline 27 & $95 \mathrm{C}-1$ & 7.00 & 6.75 & 15.28 & 17.18 & 5.21 & 6.11 & 4.61 & 4.56 & 0.30 & 0.38 & 3.23 & $2.88 *$ & 6.10 & 5.10 \\
\hline 28 & 6D-1 & 7.10 & 6.50 & 14.90 & 16.30 & 6.34 & 6.70 & $5.64 *$ & 5.15 & 0.25 & 0.29 & $3.80 *$ & $3.23 *$ & 6.20 & 5.70 \\
\hline & MEAN & 6.18 & 5.47 & 17.20 & 18.51 & 5.72 & 6.21 & 4.48 & 4.35 & 0.33 & 0.37 & 3.81 & 3.36 & 6.34 & 5.37 \\
\hline & SEm \% & 0.53 & 0.53 & 1.22 & 1.54 & 0.41 & 0.48 & 1.05 & 0.36 & 0.03 & 0.03 & 0.45 & 0.43 & 0.59 & 0.59 \\
\hline & $\mathrm{CD}$ at $5 \%$ & 1.54 & 1.53 & 3.53 & 4.46 & 1.18 & 1.39 & 1.42 & 1.05 & 0.08 & 0.10 & 1.91 & 1.82 & 1.72 & 1.71 \\
\hline & CV \% & 11.91 & 13.17 & 10.32 & 11.58 & 10.2 & 10.6 & 11.01 & 11.95 & 12.69 & 12.72 & 13.84 & 14.22 & 13.21 & 15.27 \\
\hline
\end{tabular}


Fig.1 Performance of inbreds with respect to root trait under stress condition along with checks 95C-1 and 6 D-1
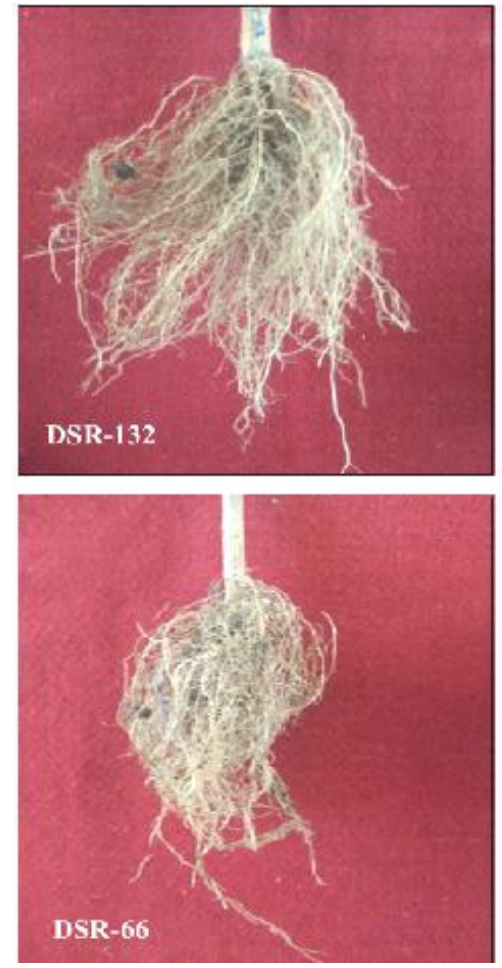
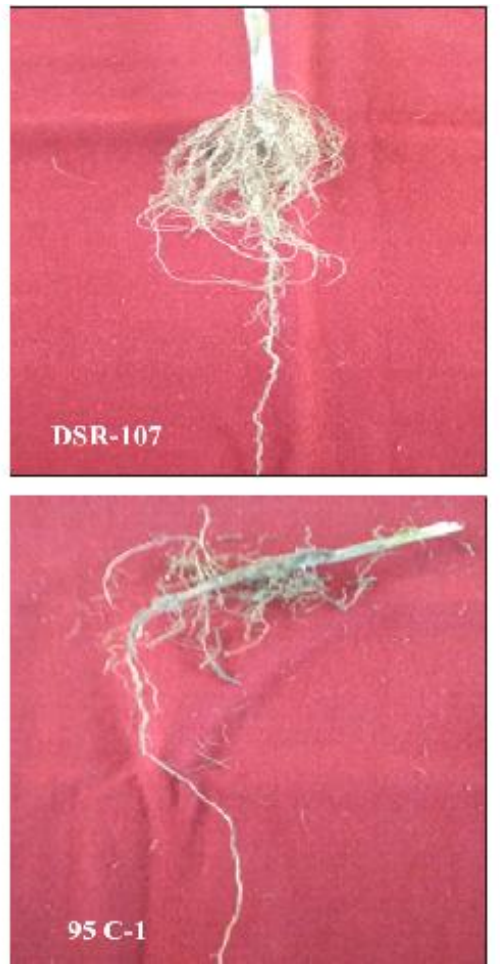
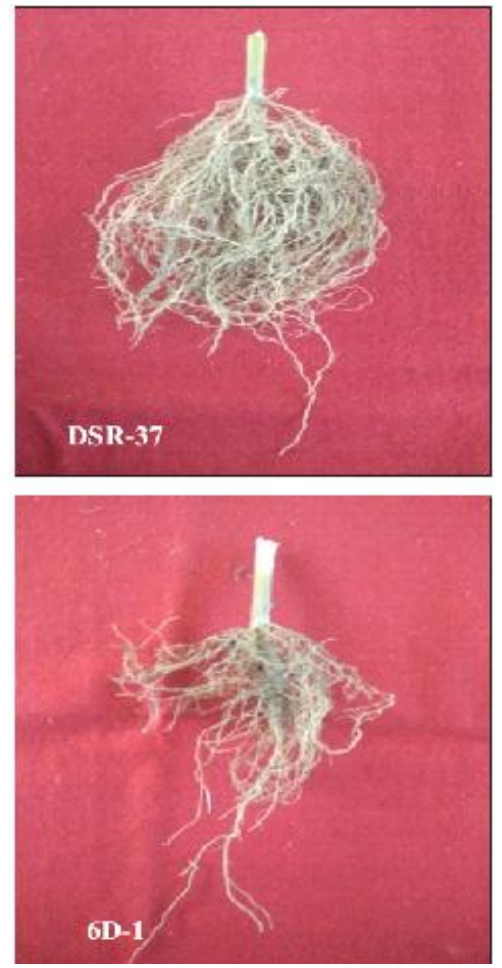

Complex quantitative trait like seed yield is highly influenced by environment. In the present investigation seed yield per plant was significantly differed under different water regimes. The maximum seed yield per plant was recorded by DSR-37 (9.5 g) and DSR-132 $(8.1 \mathrm{~g})$ under stress condition when compared to checks and other genotypes used. The head diameter was found to be higher in DSR-37 $(8.25 \mathrm{~cm})$, DSR-132 $(8.70 \mathrm{~cm})$ and DSR-66 $(8.80 \mathrm{~cm})$ when compared to all lines and checks used under stress condition. The genotypes were grouped in to high and low performing types based on performance under stress conditions for different traits, which is shown in Table 4 . The percent decrease in the yield observed was also less for DSR -37 $(5.94 \%)$ and DSR-132 (10.99\%) when compared to check $95 \mathrm{C}-1$ (16.39\%) under stress condition. Alza and Fernandez (1997) reported similar results in sunflower with less per cent reduction of yield under stress condition. Increase in the yield per plant under stress conditions was due to increase in the root length, relative water content and maintaining greenness even during stress conditions. These results were in line with experiment conducted by Hiremath and Nadaf. (2017) with inbreds DSR-14 and DSR-47 showing better performance in all traits under both conditions (Table 5 and 6).

The staygreen lines that found had the ability to grow even during stress conditions with less per cent reduction in yield and other traits, thus these inbreds can be utilized in further hybridisation programme for the development of drought tolerant OPV's and hybrids.

\section{References}

Ali, Q., Ashraf, M. and Anwar, F., 2009. Physico-chemical attributes of seed oil 
from drought stress sunflower plants. Grasas y Aceites., 60: 475-481

Aliza, J. O. and Fernandez-Martinez, J. M., 1997, Genetic analysis of yield and related traits in sunflower (Helianthus annuus L.) in dry land and irrigated environments. Euphytica., 95(2) :243251.

Chimenti, C.A., Pearson, J. and Hall, A. J., 2002. Osmotic adjustment and yield maintenance under drought in sunflower. Field Crops Res., 75: 235246

Ehab- Kheir, 2006, Assessment of genetic variability in water use efficiency, root traits and intrinsic tolerance among the cotton hybrids and cultivars. M.Sc. (Agri) thesis, University of Agricultural Sciences, Bangalore.

Geetha, A., Suresh, J. and Saidaiah, P., 2012, Study on response of sunflower (Helianthus annuus L.) genotypes for root and yield characters under water stress. Current Biotica, 6(1): 32-41.

Hiremath, G. and Nadaf, H. L., 2017, Assessment of stay green genotypes of sunflower for root traits under different soil moisture regimes. Int. J. Curr. Microbiol. App. Sci., 6(11): 11561166.

Hurd, E. A., 1974, Phenotype and drought tolerance in wheat. Agric. Meteorol.,
14: 39-55.

Rauf, S. and Sadaqat, H. A., 2008, Identification of physiological traits and genotypes combined to high achene yield in sunflower (Helianthus annuus L.) under contrasting water regimes. Aus. J. Crop Sci., 1 (1): 2330.

Richards, R. A., 1992, The effect of dwarfing genes in spring wheat in dry environments. II. Growth, water use and water use efficiency. Aust J. Agric. Res., 43: 529-539.

Satyabrata, M., Hedge, M. R. and Chattopodhay, S. B., 1988, Hand Book of Annual Oilseed Crops. Oxford IBH Pub. Co. (Pvt) Ltd. New Dehli. pp.176.

Soorninia, F., Toorchi, M., Norouzi, M. and Shakiba, M., 2012, evaluation of sunflower inbred lines under drought Stress. Universal J. Environ. Res. Technol., 2 (1): 70-76.

Skoric, D., 2009, Sunflower breeding for resistance to abiotic stresses. Helia, 32(50): 1-16.

Vikram, S. R., 2008, Development of trait introgressed sunflower (Helianthus Annuus L.) $\mathrm{F}_{1}$ hybrids and their evaluation for drought tolerance under stress full condition. M.Sc. (Agri.) Thesis, Univ. Agric. Sci. Bangalore (India).

\section{How to cite this article:}

Harshavardan J. Hilli and Shobha U. Immadi. 2019. Evaluation of Staygreen Sunflower Lines for Yield under Drought Conditions (Helianthus annuus L.). Int.J.Curr.Microbiol.App.Sci. 8(12): 1100-1110. doi: https://doi.org/10.20546/ijcmas.2019.812.139 